\title{
Recommendations for the Assessment and Management of Pre-existing Drug-Reactive Antibodies During Biotherapeutic Development
}

\author{
Li Xue, ${ }^{1,9}$ Adrienne Clements-Egan, ${ }^{2}$ Lakshmi Amaravadi, ${ }^{3}$ Mary Birchler, ${ }^{4}$ Boris Gorovits, ${ }^{1}$ Meina Liang, ${ }^{5}$ \\ Heather Myler, ${ }^{6}$ Shobha Purushothama, ${ }^{7}$ Marta Starcevic Manning, ${ }^{8}$ and Crystal Sung ${ }^{3}$
}

Received 18 May 2017; accepted 31 July 2017; published online 6 November 2017

\begin{abstract}
Anti-drug antibodies (ADA) pose a potential risk to patient safety and efficacy and are routinely monitored during clinical trials. Pre-existing drug-reactive antibodies are present in patients without prior drug exposure and are defined by their ability to bind to a component of the drug. These pre-existing drug-reactive antibodies are frequently observed and could represent an adaptive immune response of an individual who has been previously exposed to antigens with structural similarities to the biotherapeutic. Clinical consequences of these antibodies can vary from no impact to adverse effects on patient safety, exposure, and efficacy, and are highly dependent on biotherapeutic modality, disease indications, and patient demographics. This paper describes how the immunogenicity risk assessment of a biotherapeutic integrates the existence of pre-existing drug-reactive antibodies, and provides recommendations for risk-based strategies to evaluate treatment-emergent ADA responses.
\end{abstract}

KEY WORDS: anti-drug antibodies; clinical impact; pre-existing drug-reactive antibodies; risk assessment and mitigation.

\section{INTRODUCTION}

Several reports describe pre-existing reactivity from antidrug antibody (ADA) assays in baseline samples collected from treatment-naïve subjects (1-7). This pre-existing reactivity can be due to the presence of antibodies from treatment-naïve subjects or due to other non-antibody interferents. The pre-existing drug-reactive antibodies may be a part of the innate immune response of the host. Preexisting antibodies may have been arisen in response to xenobiotic environmental antigens unrelated to the drug, or due to a prior exposure to a homologous biologic drug (8). In

${ }^{1}$ Biomedicine Design, Pfizer Worldwide Research and Development, 1 Burtt Rd, Andover, Massachusetts, USA.

${ }^{2}$ Janssen Research \& Development, LLC (Johnson \& Johnson), Welsh and McKean Roads, Spring House, Pennsylvania, USA.

${ }^{3}$ Clinical Laboratory Sciences, DSAR, Sanofi R\&D, Framingham, Massachusetts, USA.

${ }^{4}$ Clinical Immunology, GlaxoSmithKline, King of Prussia, Upper Merion, Pennsylvania, USA.

${ }^{5}$ Clinical Pharmacology and DMPK, MedImmune, Mountain View, California, USA.

${ }^{6}$ Analytical and Bioanalytical Development, Bristol-Myers Squibb, Princeton, New Jersey, USA.

${ }^{7}$ UCB Celltech, Slough, UK.

${ }^{8}$ Amgen Inc., One Amgen Center Drive, Thousand Oaks, California, USA.

${ }^{9}$ To whom correspondence should be addressed. (e-mail: li.xue@pfizer.com) contrast to the ADAs that are induced or boosted upon a biotherapeutic product treatment and show binding specificity to the biotherapeutic, pre-existing drug-reactive antibodies may bind to environmental and/or pathogenic antigens that have overlapping epitopes with multiple drug products. These antibodies are components of the human autoantibody or cross-reactive antibody repertoire and may or may not be boosted upon exposure to a biotherapeutic product. In some instances, pre-existing antibodies may be directed against inert components of biotherapeutics such as polyethylene glycol (PEG) to which an individual has developed reactivity due to prior exposure by way of medicines or food products. Non-antibody interferents may include soluble targets that promote bridging of drug-derived reagents or aggregates found in labeled reagents used in the ADA assay (9-11). Several assay interference mitigation approaches have been reported to minimize non-antibody interferents that confound the detection of pre-existing drug-reactive antibodies and drug-induced ADAs $(9,12)$.

A prior survey conducted and published by the Therapeutic Product Immunogenicity Focus Group (TPIFG) of the American Association of Pharmaceutical Scientists (AAPS) indicated that despite the broad experience in encountering pre-existing drug-reactive antibodies in treatment-naïve samples, the impacts of these antibodies are still largely unknown (3). The survey revealed a variety of approaches that are currently employed to detect, characterize, and mitigate the impact of pre-existing reactivity including pre-existing antibodies in ADA assays. Herein, gaps identified in the survey 
are addressed and a harmonized definition of pre-existing antibodies is provided. Several bioanalytical recommendations are also provided for defining ADA assay cut points in the presence of pre-existing antibodies, characterizing preexisting antibodies, reporting pre-existing antibody data, assessing the impact of these pre-existing antibodies, and managing the impact of pre-existing antibodies in non-clinical and clinical settings.

\section{CLASSIFICATION (DEFINITION) OF PRE-EXISTING DRUG-REACTIVE ANTIBODIES}

Pre-existing drug-reactive antibodies can be defined as immunoglobulins reactive with protein or glycan domains on the biotherapeutic compound that are present in the matrix of subjects without prior exposure to the biotherapeutic. Preexisting antibodies of various specificities have been described including those with reactivity to PEG, glycans, microbial (bacterial, fungal, etc.) toxins, viral proteins, and cytokines (13-18). Naturally occurring antibodies to neoepitopes that are revealed after conformational change of a biotherapeutic with reactivity to the hinge region and/or other internal residues of the IgG have also been reported (19-21).

When considering the nature of pre-existing drugreactive antibodies, it is important to also consider the context in which they may exist. For example, pre-existing antibodies from naïve subjects may have different clinical consequences when compared to pre-existing antibodies from subjects that have had prior therapy with a structurally similar product. The treatment-naïve, pre-existing drug-reactive antibodies may be classified to one of the two sub-categories:

1) Autoantibodies-antibodies that are specific to selfantigens (e.g., cytokines, growth factors) or polyreactive IgGs and IgMs (e.g., rheumatoid factors $[\mathrm{RF}])$. These antibodies are normally non-neutralizing (22).

2) Cross-reactive antibodies-antibodies that may emerge due to prior exposure of environmental antigens that have molecular mimicry to a biotherapeutic or prior treatment with a product structurally similar to a biotherapeutic.

The pre-existing antibodies induced upon prior treatment with the same biotherapeutic product or products that have homologous epitopes (e.g., different versions of a recombinant protein therapeutic) are ADAs in nature. These antibodies exhibiting specificity to the prior treatment may not be components of the human autoantibody and crossreactive antibody repertoire. Nonetheless, many of the analytical strategies to address challenges of pre-existing drug binding antibody assessments in treatment-naïve subjects can apply to pre-existing antibodies in populations with prior exposure to a biotherapeutic.

\section{FACTORS TO CONSIDER WHEN EVALUATING POTENTIAL PRE-EXISTING DRUG-REACTIVE ANTIBODIES}

The ADA assay-positive signals detected from the treatment-naïve samples could be mediated by the preexisting drug-reactive antibodies as well as other non- antibody interferents. Sponsors are recommended to identify the potential risk, for example, assess the presence of soluble target, to minimize the non-antibody interference at the stage of assay development. The decision for additional preexisting antibody confirmation and characterization should be driven by the clinical relevance assessed by the sponsor. The extent and exact type of characterization applied for a given biobiotherapeutic will depend on several factors, including the stage of drug development, pre-existing antibody levels and prevalence, and structural characteristics of the biotherapeutic.

\section{Stage of Drug Development}

The stage of drug development could influence the extent of pre-existing antibody characterization that will add value to the program. For example, immune responses in non-clinical studies are not typically characterized beyond the screening assay unless there is a potential for the consequences of the immune response to influence the clinical immunogenicity assessment strategy. When it is essential to understand if ADAs are boosted in this situation, a titration method is recommended to adequately compare the magnitude of the pre-existing antibody response with the post-dose ADA response. However, it may be sufficient to rely on the magnitude of the pre- and post-dose screening results if the assay shows linearity in the range of signals observed. In this situation, the screening results of pre- and post-dose samples, coupled with the overall impact of that response on pharmacokinetic (PK) results and safety, can aid in drawing conclusions about the development of impactful ADA. In a clinical setting, it is important to understand whether the preexisting antibodies are boosted upon treatment. If only a small proportion of subjects have a pre-existing antibody response and there is no major impact on PK, post-treatment ADA status, efficacy, or safety, it may not be necessary to characterize the response beyond the screening, confirmatory, and titer assays.

\section{Pre-existing Drug-Reactive Antibody Level}

Current and emerging technology platforms used in ADA assessments are highly sensitive and therefore capable of detecting low positive assay signals. Because these assays are designed with conservative cut points, a degree of false positivity could be expected. The impact of pre-existing positive samples near the detection limits of these technology platforms is questionable. Typically, high titer assay responses in treatment-naïve subjects may be of more concern than low titer results and may warrant additional characterization.

\section{Structural Characteristics of the Biotherapeutic}

If a biotherapeutic contains multiple domains, such as a PEG domain, a protein domain that is homologous to an endogenous counterpart or domains for multiple targets, it may be important to characterize the pre-existing antibodies for their domain binding specificity. For pre-existing antibodies to biotherapeutic antibodies, mapping of the pre-existing antibody response to the Fab or Fc portion could be informative, particularly if there are unusual post- 
translational modifications or mutations in the $\mathrm{Fc}$ region of the drug. However, for most human mAb-based biotherapeutics, this is usually unnecessary. Some preexisting antibodies are elicited by the quality attributes of the biotherapeutic. Therefore, it is recommended to evaluate the biotherapeutic structure and the manufacturing processes when designing the strategy to evaluate the pre-existing drugreactive antibodies.

\section{Needs for Pre-existing Antibody Isotyping}

When pre-existing antibodies are observed, further isotype characterization of a subset of antibody-positive samples during assay development is usually not essential if the risk of pre-existing antibodies is low. Potential preexisting $\mathrm{IgE}$ responses would be of most concern, but have rarely been reported in biotherapeutic programs. One notable exception is for cetuximab, where pre-existing antiGal- $\alpha-1,3-G a l$ IgE antibodies have been described resulting in significant hypersensitivity reactions in a subset of the cetuximab-treated patients after the initial dose. The need for validation of an $\mathrm{IgE}$ isotype assay and prospective assessment of subjects was not apparent because reactions were only seen in a subset of subjects from certain geographical regions (15). While it was important to understand the mechanism behind these significant hypersensitivity reactions through isotyping the ADA responses retrospectively, the treatments for such reactions were dictated by the clinical presentation of anaphylaxis not by assessments of ADA isotypes during clinical studies. During pre-study ADA assay development, $\mathrm{IgE}$ isotype investigations in donors with pre-existing drugreactive antibodies are not recommended, unless (1) a known relationship between antibody isotypes and clinical outcomes exist for similar compounds to the biotherapeutic; (2) the biotherapeutic has moieties with high potential for preexisting antibodies (e.g., non-human glycans), and, most importantly; (3) if the knowledge of pre-existing $\operatorname{IgE}$ responses would cause unmanageable safety risks for patients, or/inform go/no go decisions for the program. In clinical studies, the application of validated isotyping (e.g., $\mathrm{IgE}$ ) assay prospectively for all pre-existing antibody-positive samples is not likely needed unless it is dictated by the clinical safety observation, and the pre-existing $\operatorname{IgE}$ levels are to be used to aid in clinical study subject enrollment or patient stratification.

In summary, a risk-based approach should be applied when determining the need for isotype analysis of preexisting antibodies and, an immune-mediated safety event should be the trigger. Unless the pre-existing antibody isotypes would impact patient safety, the pre-study validation of an isotype assay and its prospective application to preexisting antibody-positive samples in a clinical study is not warranted.

\section{Assay Strategies for Evaluating Potential Pre-existing Antibodies}

Initial evidence of the pre-existing antibodies may emerge during individual matrix testing while establishing the assay cut point value. An assay cut point value that is determined based solely on the statistical analysis of distribution of signal generated by drug-naïve samples may be greatly impacted by the presence of pre-existing drugreactive antibodies (or other factors leading to unusually strong assay response). The presence of pre-existing antibodies in a pooled sample selected as the naïve matrix control (NC) for the assay also should be understood as this can lead to an abnormal performance of the control. Samples that generate high assay signal and can be considered as outliers are typically removed from the overall data collection during statistical analysis of the data to avoid unsuitably high cut point value. However, in cases where samples with preexisting antibodies are highly abundant, a statistical evaluation may not adequately remove the influence of pre-existing antibodies on the cut point. This scenario, coupled with the fact that pre-existing antibodies are viewed as potential risk factor for unwanted clinical outcomes, emphasizes the need to distinguish pre-existing antibodies from other assay interfering matrix components.

If pre-existing reactivity is due to non-antibody interfering assay components, the impact could be mitigated by adjusting assay conditions or by substantially modifying assay reagents. For example, a multimeric drug target which may lead to a false positive ADA scoring can be removed by applying a sample pretreatment step $(10,23)$. However, ADA methods should not be adjusted to prevent the detection of pre-existing antibodies as these may resemble treatment-induced drug-specific ADA and should be correlated with clinical outcomes. In some cases, nonspecific matrix binding factors, such as rheumatoid factors (RFs) or heterophilic antibodies, may be viewed as compound-specific. Theoretically, these factors could impact the mechanism of action (MOA) of biotherapeutic antibodies in which the Fc domain plays an essential role. An assessment needs to be done based on the biotherapeutic structure and MOA prior to determining whether the assay conditions should be adjusted (e.g., by commercially available blocking reagents) to reduce signal due to these factors.

Confirmation assays are often applied to determine the specificity of pre-existing antibodies. It should be noted that a typical drug-inhibition-based confirmatory assay may not directly distinguish between drug cross-reacting ADAs and other interfering components. Alternative tests are therefore required to determine the true nature of interfering factors. Methods that can be applied to evaluate the nature of assay interfering factors include Protein $\mathrm{A} / \mathrm{G}$ depletion, RF-specific blocker, and removal or blocking of drug target (23-27). The approach based on pre-adsorption and extraction of the preexisting antibody, e.g., using the abovementioned reagents, would have the advantage of clearly distinguishing reactivity due to antibody responses versus other assay interferents. However, this could be very challenging due to sample volume requirements and variability in extraction.

Determination of the pre-existing antibody binding specificity is an important component of the pre-existing antibody characterization strategies. Pre-existing antibody characterization may facilitate prediction of potential preexisting antibody impact on product pharmacokinetics (PK), pharmacodynamics (PD), and safety. Characterization strategies should be customized in a risk-based manner and may also include evaluation of the ability of pre-existing antibodies to neutralize drug activity (neutralizing antibody [NAb] specificity), identification of immunoglobulin isotype (e.g., 
$\operatorname{IgE})$, and a more detailed semi-quantitative description of pre-existing antibody magnitude (e.g., titer analysis). Other types of characterization (e.g., affinity of binding, more detailed domain mapping) may be appropriate based on the exact nature of the molecule and risk factors. If a sponsor decides that further characterization of the pre-existing antibody response is warranted, consideration needs to be given to the samples to be selected for characterization. In most cases, all study samples with pre-existing antibodies would be selected for characterization. Alternatively, representative samples may be selected. Furthermore, the type of characterization performed should be carefully selected based on the value provided to decision-making for the drug development program. Table I summarizes some of the characterization approaches that could be undertaken along with the pros/cons/caveats of such characterization.

\section{STRATEGIES FOR PRE-EXISTING ANTIBODY CUT POINT SELECTION AND REPORTING OF IMMUNOGENICITY DATA}

\section{Determination of Assay Cut Point in the Presence of Pre- existing Antibodies}

The screening cut point is defined as the level of response of the screening assay at and above which a sample is defined to be a "reactive" for the presence of ADA, and below which it is negative (28). The presence of pre-existing antibodies may affect the determination of an appropriate screening cut point and therefore should be addressed.

Assay cut points are often relative measures presented as a ratio of sample signal to negative control signal. Selection of an appropriate negative control may be complicated by the presence of pre-existing drug-reactive antibodies, as they may be present in the drug-naïve sera used to make a negative pool. Appropriate screening of serum samples from drugnaïve individuals should be conducted before preparation of a negative pool. Statistical outliers in this population should be removed before pooling. It is important to set criteria for negative control preparation to ensure lot to lot consistency.

\section{Approaches for Determining an Assay Cut Point when Pre-existing Antibodies Are Present}

Multiple approaches may be utilized to determine the assay cut point in the presence of pre-existing antibodies. The sponsors are recommended to examine the pre-existing antibody signal level, prevalence, and distribution pattern, thereby to determine the most case-appropriate cut point selection method(s). In general, a conservative cut point that allows for the detection of all antibody positives is recommended. The following recommended approaches for mitigating the impact of pre-existing antibody-positive samples on the ADA assay cut point are provided.

- $\quad$ Elimination of pre-existing positive samples with a characterization or specificity assay for cut point determination

First, true antibody-positive samples should be identified and eliminated from the drug-naïve samples. These true antibody-positive samples can be identified by a characterization assay, such as immunodepletion. Alternatively, a practical conservative approach using a pre-established subjective \% inhibition cut point in a standard specificity inhibition assay can also eliminate pre-existing antibodypositive samples (54). Once positives are removed, the cut point should be calculated with an adequate quantity of remaining negative samples using standard approaches (i.e., 95th percentile) $(29,55)$. The presence of pre-existing antibodies may necessitate a larger sample size during assay validation so that enough antibody-negative subjects will remain for statistical assessment of the cut point. The removal of pre-existing positives from the validation data set results in a lower cut point and enables the detection of these antibodies in subsequent clinical sample analysis.

- Removal of statistical outliers in pre-existing positive samples prior to cut point determination

Depending on the prevalence and distribution of preexisting antibody-positive samples, clear delineation between positive and negative samples before a cut point is established may be difficult. The statistical outliers determined by statistical analysis of the data set are recommended to be removed from the cut point calculation. Thereafter, if the data set exhibits normal distribution, the cut point can be calculated using standard approaches (i.e., 95th percentile); if the data set exhibits abnormal distribution, the nonparametric approaches may be used as deemed appropriate.

- Creation of a pseudo ADA-negative population in the presence of high-prevalence pre-existing antibodies for cut point determination

When the pre-existing drug-reactive antibodies are present in very high prevalence, ADA-negative treatment-naïve samples are not available for cut point assessment. Inclusion of preexisting antibodies for a cut point establishment will artificially elevate the cut point, leading to false negatives for immunogenicity assessment. In order to overcome the challenge, a pseudo ADA-negative population can be generated for cut point determination during validation. In order to accomplish this, the drug may be spiked into drug-naïve samples with a sufficient concentration to completely abolish pre-existing antibody signal. The remaining signals of the samples will be used for cut point determination. This approach provides an alternative, practical solution for immunogenicity assay cut point establishment when high prevalence of pre-existing antibodies is detected in the study population (30). This approach requires optimization of the immunoinhibition conditions can be used for both ADA and NAb assay cut point assessments. However, for cell-based NAb assay, this approach can be more challenging as drug itself can directly modulate the assay readout and other reagents to inhibit pre-existing antibody effect without affecting the assay readout will be required.

- $\quad$ Titer approach used instead of a screening cut point approach to identify treatment-induced antibodies

In the cases when a screening cut point cannot be appropriately determined, for instance, owing to the high 


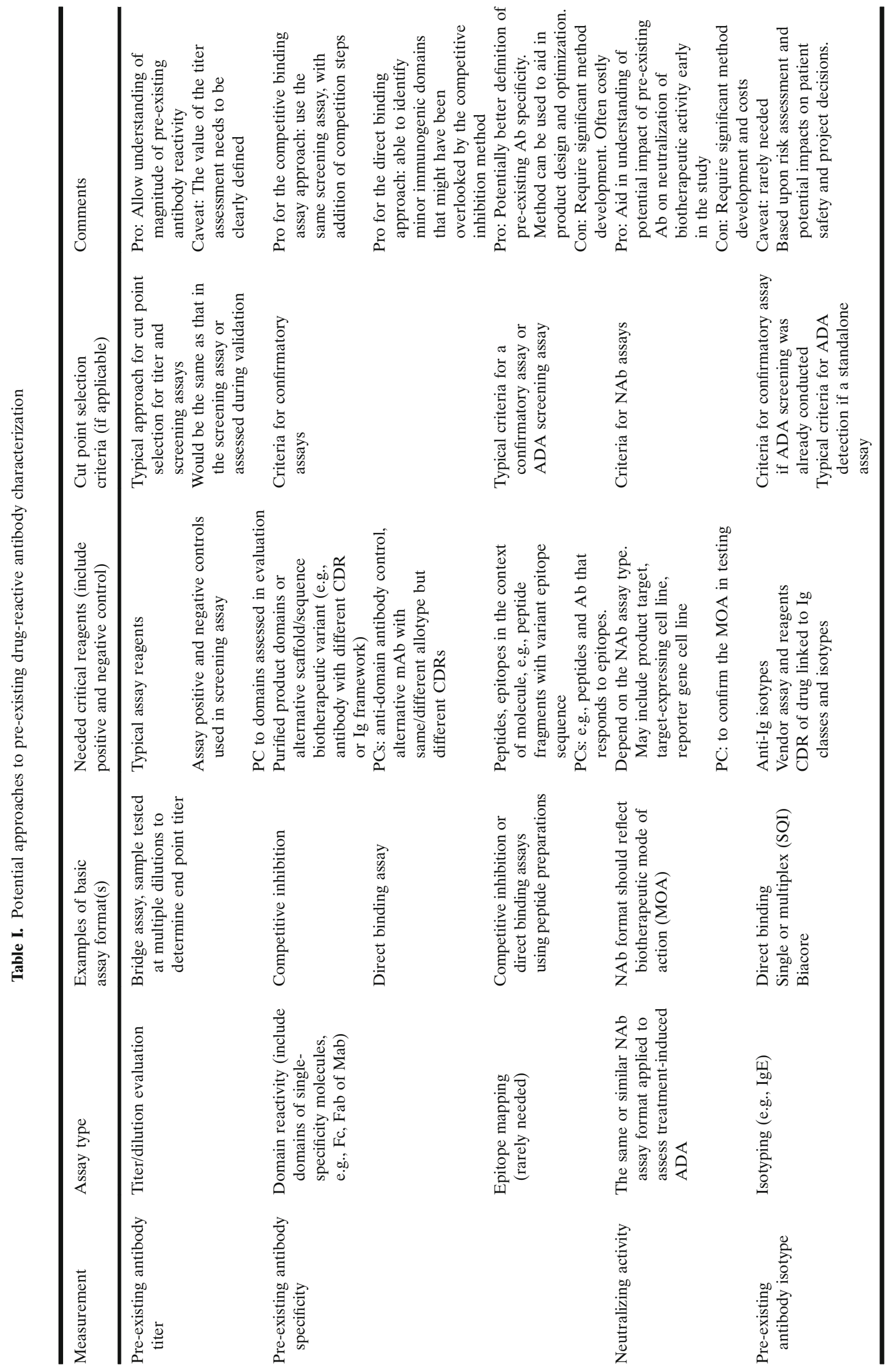


prevalence of pre-existing antibodies and the strong ADA signals in the baseline samples, a direct titter-based approach is recommended to distinguish pre-existing antibodies from treatment-induced antibodies. Treatment-induced antibodies are defined based on the titer increase in post-dose over predose samples (31).

\section{Describing Pre-existing Antibodies in Study Reports}

Pre-existing antibodies should be included in the assessment of clinical immunogenicity. As outlined above, appropriate assay cut points that allow for detection of all antibody positives, whether pre-existing or treatment induced/boosted should be employed if possible. Once all antibody-positive samples are identified, additional characterization of the antibodies may be employed and reported. Subjects positive for pre-existing antibodies should be reported as a percentage of the total number of subjects whose baseline samples were tested for ADA (prevalence of pre-existing antibodies), and the titer range of baseline positives may also be reported when available. Treatment-boosted ADA should be reported. When any one sample taken after the initial drug administration has an ADA titer that is greater than the baseline titer by a scientifically reasonable margin such as fourfold or ninefold difference when a twofold or threefold serial dilution is applied for titration, it can be considered treatment boosted (31). The titer threshold defined as "scientifically reasonable" will depend on the study and individual assay parameters, such as titer dilution and precision. It is recommended that the impact of pre-existing antibodies on treatment ADA boosting, product PK, PD, efficacy, and patient safety should be evaluated and described in study reports.

\section{ANALYSIS OF PRE-EXISTING DRUG-REACTIVE ANTIBODY IMPACT}

As with drug-induced ADAs, the clinical impact of preexisting drug-reactive antibodies varies greatly from no impact to reduced efficacy or life-threatening adverse events. The risk associated with pre-existing antibodies should be assessed in the context of the totality of the biotherapeutic development program, taking into account the product attributes, nature of pre-existing antibodies, as well as the clinical application of the biotherapeutic and the study populations. The scope of the risk assessment includes the following: (1) the likelihood of pre-existing antibody presence at baseline (in pre-dose samples); (2) the potential for analytical methodology to detect clinically meaningful preexisting antibodies versus low positive assay signal; and (3) the potential impact of pre-existing antibodies on treatmentemergent immunogenicity, PK, PD, efficacy, and patient safety.

\section{The Likelihood of Pre-existing Antibody Presence at Baseline}

The prevalence of the biotherapeutic reactive preexisting antibodies can be measured by employing analytical assays for the assessment of immunogenicity in baseline clinical samples. However, in some cases and prior to the analytical measurement, the potential for detecting impactful pre-existing antibodies in the treatment-naïve subjects may be expected based upon the biotherapeutic modalities, molecular homology to environmental antigens, known prior exposure level to homologous antigens, and the immune status of the patient populations. Recombinant proteins (e.g., cytokines, growth factors), pegylated proteins, engineered antibody scaffolds, gene therapy vectors (e.g., AAV), and glycan epitopes uncommon to humans are among the product molecular attributes that are known to be associated with high prevalence of pre-existing antibodies. For a given product, the level of pre-existing antibody reactivity is likely to vary with different patient populations. For instance, patients with rheumatoid arthritis (RA) and other autoimmune diseases tend to possess larger repertoires of autoantibodies than healthy individuals, and as such, higher prevalence of pre-existing antibodies, e.g., reactive to some novel antibody scaffolds, is observed more often in RA patients than in healthy volunteers (7).

\section{The Potential to Detect Clinically Meaningful Pre-existing Antibodies}

Regardless of the clinical outcome, the presence of preexisting antibodies at baseline imposes an analytical burden that requires improved assay strategies to (1) establish appropriate cut point which allows for detection of preexisting antibodies and (2) discern the treatment-induced ADA response from the pre-existing antibodies.

While it is clear that in some cases pre-existing antibodies have the potential to impact safety and efficacy of a drug product, they may not have any impact in other cases (3). Often times, a very low-level signal that is detected in the predose subjects may be due to analytical and/or biological noise as opposed to a true positive result that warrants further investigation.

There have been significant technological advances in analytical platforms to enable development of highly sensitive and robust immunogenicity assays. To differentiate an ADApositive versus an ADA-negative sample, it is standard in industry to develop an assay cut point by employing statistical methods based on the distribution of assay signals from a drug naïve population. This approach, together with the newer highly precise assay detection platforms (i.e., with very low analytical variability), routinely leads to very low cut points near the sensitivity limits of the instrument that may not be meaningful across a more extensive application of an assay. While it is important to implement sensitive assays to detect immunogenicity of biotherapeutic proteins, this strategy may lead to a large fraction of pre-dose clinical samples classified as positive for pre-existing antibodies, even if they only exhibit a very weak signal above the cut point. It is possible that low level of antibodies may exist in the clinical subjects that may appear barely above the assay threshold and become detectable due to the nature of the highly sensitive assay platform. However, it is important for the researchers to pay close attention to the clinical immunogenicity data and interpret the data in the context of the prestudy validation. In cases where the in-study population differs from populations used during validation cut point setting, an in-study cut point may need to be set. In some 
cases, a meaningful pre-study or in-study cut point may not be achievable when there is a high prevalence of pre-existing antibodies with high signal. In this case, setting a threshold of difference in titers between pre-existing and treatmentboosted ADA responses is highly recommended. The strategy used to identify pre-existing antibodies and treatmentboosted ADA responses must be informed by the totality of evidence provided by the pre-study validation experiment results, the assessment of clinical study baseline samples, the assay technology, and the known pre-existing antibody risk factors of the biotherapeutic.

Functional Impact on Treatment-Emergent Immunogenicity, PK, PD, Efficacy, and Patient Safety

As with the prevalence of pre-existing antibodies, the potential functional impact of pre-existing antibodies is diversified and likely connected with the biotherapeutic modalities, components of manufacturing and formulation, and patient populations. Prior to a clinical study, an assessment of the nature and specificity of pre-existing antibodies, if detected during pre-study assay development, may aid in the prediction of their clinical impact. Although it is highly challenging to predict the ultimate clinical consequences of these antibodies, it is important to evaluate their impact on treatment-induced ADA boosting, PK, PD, efficacy, and/or patient safety during a clinical study (31). If pre-existing antibodies are autoreactive antibodies specific to self-antigens (e.g., cytokines, growth factors) or are polyreactive IgGs and IgMs (e.g., $\mathrm{RF}$ ), they may not impact PK/PD or clinical outcomes because they are normally non-neutralizing and tolerated in humans. However, in the events of tolerance breakage, autoantibodies may undergo affinity maturation and epitope spreading, leading to boosted ADA response post-treatment and neutralization of the administered recombinant protein product. Loss of efficacy or accelerated disease state is also an expected outcome (32,33). Therefore, a risk-based approach is recommended to define the degree and the scope of the pre-existing antibody impact analysis (based on the PK/PD and clinical observations).

Pre-existing antibodies reactive to gene therapy vectors, pegylated proteins, glycan epitopes, or biotherapeutic antibodies are likely to fall within the category of cross-reactive pre-existing antibodies. To aid in determining the risk of preexisting antibodies for a biotherapeutic program, different scenarios that describe the root causes and attributes of preexisting antibodies are shown below:

- Pre-existing antibodies to antibody-based biotherapeutics

For antibody-based biotherapeutics, the binding specificity of the pre-existing antibodies is likely to be a determinant of their overall immunogenicity risk profiles. Pre-existing antibodies that recognize the idiotopes are expected to interfere with target binding when the idiotopes are part of the antigen binding site, or in close proximity to the antigen binding site. These antibodies may lead to drug neutralization, loss of efficacy, and ultimately treatment failure $(20,34)$. Pre-existing antibodies recognizing the allotopes of antibody biotherapeutics are less likely to neutralize the interaction between the biotherapeutic and the target, therefore, less likely to cause rapid clearance of the drug or have major impact on efficacy (35). It is theoretically possible for non- neutralizing pre-existing antibodies to mediate downstream effects via crosslinking the biotherapeutic target. It is possible that pre-existing antibodies against a biotherapeutic antibody exhibit mixed binding specificity for both idiotopes and allotopes.

\section{- $\quad$ Pre-existing antibodies to PEGylated drugs}

Pre-existing antibodies to polyethylene glycol (PEG) are reported in various frequencies dependent on the level of prior exposure to PEG-containing commodities or medical procedures, as well as the analytical methods used for pre-existing antibody detection (16,36-39). While a high prevalence of anti-PEG preexisting antibodies was not associated with impaired response to PEG-interferon in hepatitis $\mathrm{C}$ patients, increased drug clearance, reduced efficacy, and increased infusion reactions have been reported for pegloticase (PEG-porcine uricase) in patients with refractory chronic gout $(14,40)$. Additionally, pre-existing antiPEG antibodies were associated with severe immediate allergic reactions to pegnivacogin, a PEGylated aptamer in a phase $2 \mathrm{~b}$ clinical trial in patients with acute coronary syndrome $(41,42)$. Monitoring of the clinical impact of anti-PEG pre-existing antibodies is warranted on a case by case basis. If a bridging format ADA assay was used to measure antibodies against the PEGylated protein conjugate in clinic, further characterization of the pre-existing antibodies is required to determine the binding specificity for the PEG or/and the protein moiety of the conjugate. The mechanisms underlining the distinctive clinical outcomes of the anti-PEG pre-existing antibodies remain to be elucidated. The size of the protein and the way PEG molecules are conjugated to the protein moiety might be contributing factors. In addition, the potential risk of pre-existing anti-PEG antibodies to cause epitope spreading and thereby the enhanced antibody response to the protein moiety of the PEG conjugates remains to be determined.

- $\quad$ Pre-existing antibodies to viral components used in gene therapy

The prevalence of the pre-existing antibodies to gene therapy vectors is relevant to the viral serotype and the geographic locations of the patient population. Pre-existing antibodies to viral vectors can impact the efficacy of the gene therapy. The presence of neutralizing pre-existing antibodies against viral capsid proteins can block entry of the agents into targeted cells and preclude successful gene expression. For instance, the efficacy of adeno-associated virus (AAV)-based gene therapy can be impaired by the presence of neutralizing pre-existing antibodies to AAV proteins, of which anti-AAV2 serotype Abs were most prevalent across different regions, followed by antibodies to AAV1, AAV7, and AAV8 (8). A high prevalence of pre-existing antibodies to lentivirus has not been reported. By avoiding the inhibition by the neutralizing antiviral vector pre-existing antibodies in the peripheral blood, the gene therapies administered into target tissues are of lesser concern than the systematically administered gene therapies.

- Pre-existing antibodies to enzyme/protein replacement therapies

Pre-existing antibodies to protein replacement therapies may be attributed to exposure to structurally similar or mutated endogenous proteins. As replacement therapy in general is a long-term therapy, it should be noted that 
patients may potentially undergo product switch during the lifetime of treatment. Therefore, the development of preexisting antibodies against specific replacement proteins is not surprising. The potential impact of these pre-existing antibodies is largely unknown, though, in some cases, these preexisting antibodies could have serious consequences if they can render the therapy ineffective leading to clinical deficiency type syndrome $(18,43)$. Natural induction of immune tolerance after long-term enzyme replacement therapy (ERT) in mucopolosaccharidosis I patients with pre-existing antibodies was reported (18). The ability to induce immune tolerance in response to long-term ERTs and being maintained over the course of treatment warrants further investigation. In patients with Pompe disease, pre-existing antibodies to human recombinant GAA did not correlate with adverse effects or otherwise impact on clinical outcome of the treatment (44). Similarly, the pre-existing antibodies to other endogenous proteins either have no apparent effect on clinical events or the effect is unclear that requires evaluation on a case by case basis $(45,46)$. As many endogenous proteins are biologically redundant, neutralization of biotherapeutic proteins by pre-existing antibodies may not produce significant clinical deficiency syndromes for all biotherapeutic proteins with endogenous counterparts. However, caution needs to be taken as not all biological functions of an endogenous protein are well characterized or understood.

\section{- $\quad$ Pre-existing antibodies to non-human glycans}

IgE-type pre-existing antibodies may result in hypersensitivity reactions in patients receiving treatment. For example, the $\mathrm{IgE}$ isotypes of anti-galactose-1,3-galactose pre-existing antibodies, but not the IgG isotypes, have been shown to cause severe anaphylactic reactions in patients treated with cetuximab $(15,47,48)$. In another example, $\mathrm{N}$-linked glycans present in plant-derived biotherapeutic glycoproteins are known to crossreact with pre-existing antibodies ( $\mathrm{IgE}, \mathrm{IgG} 4, \mathrm{IgG} 1)$. However, their relevance to hypersensitivity in patients who receive the biotherapeutic treatment is not yet well defined and possibly varies on a case by case basis (48-52).

- $\quad$ Pre-existing antibodies due to prior biologic exposure

Pre-existing drug-reactive antibodies may also develop due to prior treatment with the products in the same class. The ADAs developed upon treatment with an earlier therapy may appear pre-existing to the subsequent treatment with another drug that has homologous epitopes (e.g., multiple versions of interferons and erythropoietins). In this scenario, a potential for boosted anti-drug antibody titers may exist due to memory responses.

\section{PRE-EXISTING ANTIBODY MANAGEMENT IN CLINICAL AND NON-CLINICAL SETTINGS}

The risk of the clinical impact due to pre-existing antibodies should be assessed early in biotherapeutic development. In cases where the clinical relevance of pre-existing drug-reactive antibodies is anticipated, a proactive approach to mitigate the risk potential may aid in designing the most meaningful and efficient non-clinical and clinical studies, for instances, implementing pre-existing antibody screening for patient enrollment or employing immune mitigation strategies. One aspect of a fit for purpose risk mitigation strategy is to minimize, or at least thoroughly understand, the potential impacts of pre-existing antibodies on the accuracy of PK and ADA analytical assays and to devise an appropriate method to report reliable $\mathrm{PK}$ and ADA study results. The most important aspect of a risk mitigation strategy, however, is to alleviate the potential adverse impact of pre-existing antibodies on patient safety, which should be reflected in the study design and patient stratification criteria.

\section{Management of Pre-existing Drug-Reactive Antibody Risk Impact in the Pre-clinical Setting}

The proactive risk mitigation and management of symptoms from pre-existing drug-reactive antibodies in a non-clinical species is generally not necessary as the immunogenicity response of animals is generally not translated to humans.

However, when a high prevalence and high titer of preexisting antibodies are expected in the study animals and imbalance of pre-existing antibody-positive animals from different treatment groups may lead to misinterpretation of study results, it is imperative to manage the impact of preexisting antibodies in the study. For example, an approach was successfully implemented in a non-clinical PK-PD study designed to evaluate comparability of various lot materials produced by different processes (53). In this case, pre-existing antibodies observed in study animals were a variable that could lead to inaccuracy in PK-PD comparability assessment. To mitigate the risk, animals were pre-screened for the presence of pre-existing antibodies. Only the animals with low pre-existing antibody signal were selected for the study since it was not practical to exclude all pre-existing antibodypositive animals from the study due to high prevalence. In addition, the selected animals were randomized for treatment assignment based on pre-existing antibody signals. Although this approach is practical and acceptable for a PK-PD comparability study, it may not be appropriate for a safety study intended to evaluate safety for a broader population rather than the pre-existing antibody-negative population. Nonetheless, if feasible, the distribution of animals for various treatment cohorts based on pre-existing antibody status (or pre-existing antibody levels) may be an appropriate study design, especially when high prevalence is observed.

Early estimates of the clinical prevalence of pre-existing antibodies can be obtained by conducting early screening using commercial samples or baseline/placebo samples from prior or related clinical studies representative of the intended clinical population. When warranted, identification of the preexisting antibody specificity in these samples may (1) early inform the potential clinical impact and the corresponding mitigation strategies and (2) help improve the clinical ADA and PK assay strategies for clinical sample analysis. Furthermore, in certain cases, the pre-existing antibody specificity may be translatable from animals (e.g., non-human primates) to humans. Therefore, prior knowledge of pre-existing antibody prevalence, specificity or impact in both species 
can be utilized to predict the clinical risk of the pre-existing antibodies for concurrent or next-generation biotherapeutics.

\section{Management of Pre-existing Drug-Reactive Antibody Risk Impact in the Clinical Setting: General Considerations}

In clinical studies, a number of approaches may be used for managing the impact of pre-existing drug-reactive antibodies, each with advantages and limitations. Commonly, the presence of pre-existing antibodies is assessed before treatment and is not used as an exclusion criterion for subject enrollment in clinical studies. As such, the impact of pre-existing antibodies on treatment-emergent ADA, PK, PD, efficacy, and safety should be evaluated systematically in the study. The data may be useful for informing dose selection and late-stage clinical study design.

Because a limited set of individual matrix samples is commonly assessed during an initial ADA assay validation procedure, it is important to re-evaluate the suitability of the assay cut point value based on the observed prevalence of preexisting antibody-positive samples in baseline-incurred study samples. Ideally, the prevalence of pre-existing positive study samples at baseline should be similar to that observed during assay validation when testing drug-naïve samples. If a significant discrepancy is observed, it is recommended to consider reevaluation of the ADA assay cut point either by utilizing data obtained for baseline study samples or by obtaining additional study population-relevant drug-naïve commercial samples.

In addition to a potential implication related to treatment-induced ADA detection and risks, pre-existing antibodies may impact the ability to detect the desired drug analyte in the PK assay, or they may directly alter the rate of drug clearance $(33,34,40)$. Specifically, pre-existing antibodies may interfere with the PK assay, leading to under- or overreporting of drug concentration. An impact on drug concentration measurement can potentially confound the interpretation of PK data, especially in cases where the study population may contain varying degrees of pre-existing antibodies. The need to characterize the specificity of preexisting antibodies is therefore highlighted if a particular type of interaction is expected to affect PK assay performance. ADA, as well as pre-existing antibodies, may not only interfere but also directly enhance drug clearance by forming immune complexes. In order to better understand the specific nature of the ADA impact, including pre-existing antibodies, on drug PK, an additional investigation may be required, as previously described $(54,55)$.

In certain cases, the presence of pre-existing antibodies may be used as a criterion for study enrollment, exclusion, or study cohort assignment. This may not be advantageous as the data will not be available for evaluation of pre-existing antibody impact on treatment-boosted ADA or the impact on safety, efficacy, PK, or PD results. Another disadvantage of basing study enrollment decisions on pre-existing antibody status is that bioanalytical burden and logistic challenges for the trial increases substantially. If patient stratification is needed, a pre-existing antibody detection method will need to be developed as a companion diagnostic, significantly increasing the developmental cost. For biosimilar programs, comparability studies are ideally to be conducted in patients precluded from prior originator treatment to minimize the influences of ADAs that are specifically induced by the originator, but appear to be the pre-existing antibodies to the biosimilars. In phase III studies, when treatment-naïve subjects are rarely available, pre-screening patients for the presence of pre-existing antibodies and thereby balancing the treatment arms with equal numbers of pre-existing antibodypositive subjects and equivalent antibody titers could be a beneficial approach. In the cases of gene therapy, pre-existing antibodies to a virus that is related to a vector used in gene transduction might impact the efficacy and potential safety of gene therapy. In order to increase the probability of biotherapeutic efficacy and/or safety during drug development, pre-screening/titering results of the pre-existing NAbs can be used to determine the clinical threshold for successful gene therapy. Samples with NAb titers above the threshold may be excluded from enrollment to reduce the potential adverse impact. Clinical management for gene therapy to overcome pre-existing NAb challenge also includes plasmapheresis and saline flushing prior to viral vector administration, and induction of immune suppression.

\section{Label Considerations}

The goal of an immunogenicity assessment during a drug development program is to comprehensively evaluate and understand the impact of ADAs on patient safety and efficacy. After demonstrating a favorable benefit/risk profile and drug approval, the immunogenicity incidence is reported on the label (package insert) along with other relevant information of the drug. The purpose of the reported information is to inform the practicing physicians about the risks and benefits of the drug. Due to the complexities of analytics, assays, and cut points described above, it is important for the immunogenicity assay scientist to be closely engaged with the safety clinician and regulators to interpret the immunogenicity data and recommend appropriate language for the label (depending on the outcome of the analysis). A thorough analysis of both pre-existing and treatment-boosted responses should be included in the clinical study reports (56). We propose that in the immunogenicity section of the label, only drug-induced ADA should be reported. In cases where pre-existing antibodies demonstrate a clinical consequence, the prevalence of pre-existing antibodies along with clinical impact should also be included in the label.

\section{SUMMARY}

The FDA mentions pre-existing antibodies in five sections of the Guidance for Industry on Assay Development and Validation for Immunogenicity Testing of Therapeutic Protein Products, with special emphasis on the impact to assay cut point, considerations for assay development, titer determination, obtaining patient baseline samples, understanding the degree of pre-existing reactivity, and the potential implications pre-existing antibodies might have on patient safety and efficacy $(57,58)$. This paper provides specific recommendations on how to address these concerns in the context of immunogenicity risk assessments.

Immunogenicity risk assessments for biotherapeutics should include the potential for the existence and impact of pre-existing drug-reactive antibodies. Reactivity of treatment-naïve patient samples should be evaluated during method development and, depending on the assessed clinical risk, should be appropriately 
characterized when warranted. The clinical significance of the pre-existing antibodies in the treatment-naïve subjects varies from no impact to adverse impact on patient safety and treatment efficacy. The pre-existing antibodies developed upon prior biotherapeutic treatment may present heightened immunogenicity risk for the patients to receive the follow on or the alternative treatment with biotherapeutics that have homologous epitopes.

It is recommended that a comprehensive immunogenicity risk assessment is performed early and re-iteratively in biotherapeutic development and that pre-existing antibodies are assessed as part of that risk assessment for biotherapeutic products. The evaluation of pre-existing drug-reactive antibodies and the strategies should be linked to the clinical relevance. It is essential to understand whether the observed pre-existing antibodies have an association with the clinical safety and efficacy event. The needs and scope of the pre-existing antibody characterization driven by the clinical impact analysis should be conducted on a case-by-case and fit-for-purpose basis. The clinical significance of the pre-existing drug-reactive antibodies is likely to be related to the biotherapeutic modalities, molecular and product quality attributes as well as the patient populations and clinical applications. Although, the ultimate clinical relevance of immunogenicity data can only be determined when they are integrated with PK, safety, and efficacy data, early prediction of the pre-existing antibody impact on treatment ADA boosting, efficacy, and patient safety may be possible, for example, when established scholarship or experiences available for specific product modalities or pre-existing antibody specificities. Furthermore, the pre-existing antibody management strategies may be employed, when appropriate, to mitigate their potential impacts in the non-clinical and clinical studies.

Finally, the paper emphasizes that pre-existing drug-reactive antibody prevalence should be described in clinical reports. In general, pre-existing antibody titers should be examined in relation to post-treatment antibody titers to understand treatment ADA "boosting." If pre-existing drug-reactive antibodies are shown to have an impact on biotherapeutic safety and/or efficacy, an adequate description of their prevalence and clinical relevance should be included in the product label.

\section{ACKNOWLEDGEMENTS}

A draft manuscript was posted on the AAPS website for public feedback. The manuscript was revised according to the public feedback, reviews by the TPIFG steering committee members and the AAPS biotech leadership. The authors would like to thank all those who reviewed the manuscript and provided feedback.

\section{FUNDING INFORMATION}

This work was sponsored by the Therapeutic Product Immunogenicity Focus Group (TPIFG) of the Biotech Section, American Association of Pharmaceutical Scientists (AAPS).

\section{COMPLIANCE WITH ETHICAL STANDARDS}

Disclaimer The contents of this article reflect the personal opinions of the authors and may not represent the official positions or perspectives of their affiliated organizations.
Open Access This article is distributed under the terms of the Creative Commons Attribution 4.0 International License (http://creativecommons.org/licenses/by/4.0/), which permits unrestricted use, distribution, and reproduction in any medium, provided you give appropriate credit to the original author(s) and the source, provide a link to the Creative Commons license, and indicate if changes were made.

\section{REFERENCES}

1. Turano A, et al. Natural human antibodies to gamma interferon interfere with the immunomodulating activity of the lymphokine. Proc Natl Acad Sci U S A. 1992;89(10):4447-51.

2. Caruso A, Turano A. Natural antibodies to interferon-gamma. Biotherapy. 1997;10(1):29-37.

3. Xue L, et al. Pre-existing biotherapeutic-reactive antibodies: survey results within the American Association of Pharmaceutical Scientists. AAPS J. 2013;15(3):852-5.

4. Bendtzen K. Autoantibodies to cytokines. Eur J Clin Investig. 1998;28(4):300-1.

5. Soos JM, et al. Identification of natural antibodies to interleukin-18 in the sera of normal humans and three nonhuman primate species. Clin Immunol. 2003;109(2):188-96.

6. Sauerborn M, Schellekens H. B-1 cells and naturally occurring antibodies: influencing the immunogenicity of recombinant human therapeutic proteins? Curr Opin Biotechnol. 2009;20(6):715-21.

7. Xue L, Rup B. Evaluation of pre-existing antibody presence as a risk factor for posttreatment anti-drug antibody induction: analysis of human clinical study data for multiple biotherapeutics. AAPS J. 2013;15(3):893-6.

8. Gorovits B, et al. Pre-existing antibody: biotherapeutic modality-based review. AAPS J. 2016;18(2):311-20.

9. Chen K, et al. False-positive immunogenicity responses are caused by $\mathrm{CD} 20+\mathrm{B}$ cell membrane fragments in an anti-ofatumumab antibody bridging assay. J Immunol Methods. 2013;394(1-2):22-31.

10. Carrasco-Triguero $\mathrm{M}$, et al. Overcoming soluble target interference in an anti-therapeutic antibody screening assay for an antibody-drug conjugate therapeutic. Bioanalysis. 2012;4(16):2013-26.

11. Schwickart $\mathbf{M}$, et al. Identification and elimination of targetrelated matrix interference in a neutralizing anti-drug antibody assay. J Immunol Methods. 2014;403(1-2):52-61.

12. Ramsland PA, et al. Interference of rheumatoid factor activity by aspartame, a dipeptide methyl ester. J Mol Recognit. 1999;12(4):249-57.

13. Sorensen PS, Koch-Henriksen N, Jensen PE. Neutralizing antibodies against interferon-beta do not predispose antibodies against natalizumab. Neurology. 2011;76(8):759-60.

14. Myler H, et al. Anti-PEG antibody bioanalysis: a clinical case study with PEG-IFN-lambda-1a and PEG-IFN-alpha2a in naive patients. Bioanalysis. 2015;7(9):1093-106.

15. Chung $\mathrm{CH}$, et al. Cetuximab-induced anaphylaxis and $\operatorname{IgE}$ specific for galactose-alpha-1,3-galactose. N Engl J Med. 2008;358(11):1109-17.

16. Tillmann $\mathrm{H}$, et al. 307 High prevalence of pre-existing antibodies against polyethylene glycol (PEG) in hepatitis C (HCV) patients which is not associated with impaired response to PEGinterferon. J Hepatol. 2010;52:S129.

17. Ward BJ, et al. Human antibody response to N-glycans present on plant-made influenza virus-like particle (VLP) vaccines. Vaccine. 2014;32(46):6098-106.

18. Kakavanos R, et al. Immune tolerance after long-term enzyme-replacement therapy among patients who have mucopolysaccharidosis I. Lancet. 2003;361(9369):1608-13.

19. Brezski RJ, Knight DM, Jordan RE. The origins, specificity, and potential biological relevance of human anti-IgG hinge autoantibodies. Sci World J. 2011;11:1153-67.

20. Holland MC, et al. Autoantibodies to variable heavy (VH) chain Ig sequences in humans impact the safety and clinical 
pharmacology of a VH domain antibody antagonist of TNFalpha receptor 1. J Clin Immunol. 2013;33(7):1192-203.

21. Ruppel J, et al. Preexisting antibodies to an $F\left(a^{\prime}\right) 2$ antibody therapeutic and novel method for immunogenicity assessment. J Immunol Res. 2016;2016:2921758.

22. Elkon K, Casali P. Nature and functions of autoantibodies. Nat Clin Pract Rheumatol. 2008;4(9):491-8.

23. Gorovits B, et al. Protein-based matrix interferences in ligandbinding assays. Bioanalysis. 2014;6(8):1131-40.

24. Zoghbi J, et al. A breakthrough novel method to resolve the drug and target interference problem in immunogenicity assays. J Immunol Methods. 2015;426:62-9.

25. Rispens $\mathrm{T}$, et al. Drug interference in immunogenicity assays depends on valency. J Pharm Biomed Anal. 2013;85:179-85.

26. Patton A, et al. An acid dissociation bridging ELISA for detection of antibodies directed against therapeutic proteins in the presence of antigen. J Immunol Methods. 2005;304(12):189-95.

27. Zhong ZD, et al. Identification and inhibition of drug target interference in immunogenicity assays. J Immunol Methods. 2010;355(1-2):21-8.

28. Shankar G, Devanarayan V, Amaravadi L, Barrett YC, Bowshere R, Finco-Kent D, et al. Recommendations for the validation of immunoassays used for detection of host antibodies against biotechnology products. J Pharm Biomed Anal. 2008:1267-81.

29. Klakamp SL, et al. Application of analytical detection concepts to immunogenicity testing. Anal Chem. 2007;79(21):8176-84.

30. Schneider AK, et al. An immunoinhibition approach to overcome the impact of pre-existing antibodies on cut point establishment for immunogenicity assessment of moxetumomab pasudotox. J Immunol Methods. 2016;435:68-76.

31. Shankar G, et al. Assessment and reporting of the clinical immunogenicity of therapeutic proteins and peptidesharmonized terminology and tactical recommendations. AAPS J. 2014;16(4):658-73.

32. Hershfield MS, et al. Induced and pre-existing anti-polyethylene glycol antibody in a trial of every 3-week dosing of pegloticase for refractory gout, including in organ transplant recipients. Arthritis Res Ther. 2014;16(2):R63.

33. Steenholdt C, et al. Pre-existing IgG antibodies cross-reacting with the Fab region of infliximab predict efficacy and safety of infliximab therapy in inflammatory bowel disease. Aliment Pharmacol Ther. 2013;37(12):1172-83.

34. van Schie KA, Wolbink GJ, Rispens T. Cross-reactive and preexisting antibodies to therapeutic antibodies-effects on treatment and immunogenicity. MAbs. 2015;7(4):662-71.

35. Tatarewicz SM, et al. Epitope characterization of pre-existing and developing antibodies to an aglycosylated monoclonal antibody therapeutic of G1m17,1 allotype. J Immunol Methods. 2012;382(1-2):93-100.

36. Richter AW, Akerblom E. Polyethylene glycol reactive antibodies in man: titer distribution in allergic patients treated with monomethoxy polyethylene glycol modified allergens or placebo, and in healthy blood donors. Int Arch Allergy Appl Immunol. 1984;74(1):36-9.

37. Bell EA, Wall GC. Pediatric constipation therapy using guidelines and polyethylene glycol 3350. Ann Pharmacother. 2004;38(4):686-93.

38. Pashankar DS, Uc A, Bishop WP. Polyethylene glycol 3350 without electrolytes: a new safe, effective, and palatable bowel preparation for colonoscopy in children. J Pediatr. 2004;144(3):358-62.

39. White JT, et al. Incidence, characterization, and clinical impact analysis of peginterferon beta1a immunogenicity in patients with multiple sclerosis in the ADVANCE trial. Ther Adv Neurol Disord. 2016;9(4):239-49.
40. Zhang $\mathrm{C}$, et al. Impact of large aggregated uricases and PEG diol on accelerated blood clearance of PEGylated canine uricase. PLoS One. 2012;7(6):e39659.

41. Povsic TJ, et al. Pre-existing anti-PEG antibodies are associated with severe immediate allergic reactions to pegnivacogin, a PEGylated aptamer. J Allergy Clin Immunol. 2016;138(6):1712-5.

42. Ganson NJ, et al. Pre-existing anti-polyethylene glycol antibody linked to first-exposure allergic reactions to pegnivacogin, a PEGylated RNA aptamer. J Allergy Clin Immunol. 2016;137(5):1610-3. e7

43. Brooks DA. Immune response to enzyme replacement therapy in lysosomal storage disorder patients and animal models. Mol Genet Metab. 1999;68(2):268-75.

44. Messinger YH, et al. Successful immune tolerance induction to enzyme replacement therapy in CRIM-negative infantile Pompe disease. Genet Med. 2012;14(1):135-42.

45. Cugno $\mathrm{M}$, et al. Autoantibodies to coagulation factors: from pathophysiology to diagnosis and therapy. Autoimmun Rev. 2014;13(1):40-8.

46. Hamilton RG, et al. Interference of thrombin in immunological assays for hirudin specific antibodies. J Immunol Methods. 2012;381(1-2):50-8.

47. Jin C, et al. A plant-derived human monoclonal antibody induces an anti-carbohydrate immune response in rabbits. Glycobiology. 2008;18(3):235-41.

48. Gomord V, et al. Biopharmaceutical production in plants: problems, solutions and opportunities. Trends Biotechnol. 2005;23(11):559-65.

49. Aalberse RC, van Ree R. Cross-reactive carbohydrate determinants. Monogr Allergy. 1996;32:78-83.

50. Bencurova $\mathrm{M}$, et al. Specificity of $\mathrm{IgG}$ and $\mathrm{IgE}$ antibodies against plant and insect glycoprotein glycans determined with artificial glycoforms of human transferrin. Glycobiology. 2004;14(5):457-66.

51. Mari A. IgE to cross-reactive carbohydrate determinants: analysis of the distribution and appraisal of the in vivo and in vitro reactivity. Int Arch Allergy Immunol. 2002;129(4):28695.

52. van Ree R, Aalberse RC. Demonstration of carbohydratespecific immunoglobulin G4 antibodies in sera of patients receiving grass pollen immunotherapy. Int Arch Allergy Immunol. 1995;106(2):146-8.

53. Wang B, et al. Pharmacokinetic and pharmacodynamic comparability study of moxetumomab pasudotox, an immunotoxin targeting CD22, in cynomolgus monkeys. J Pharm Sci. 2013;102(1):250-61.

54. Kelley $\mathrm{M}$, et al. Theoretical considerations and practical approaches to address the effect of anti-drug antibody (ADA) on quantification of biotherapeutics in circulation. AAPS J. 2013;15(3):646-58.

55. Sailstad JM, et al. A white paper-consensus and recommendations of a global harmonization team on assessing the impact of immunogenicity on pharmacokinetic measurements. AAPS J. 2014;16(3):488-98.

56. Shankar G, et al. The quintessence of immunogenicity reporting for biotherapeutics. Nat Biotechnol. 2015;33(4):334-6.

57. Assay development and validation for immunogenicity testing of therapeutic protein products. Guidance for industry, FDA draft guidance April 2016 https://www.fda.gov/downloads/ Drugs/Guidances/UCM192750.pdf.

58. Guideline on Immunogenicity assessment of biotechnologyderived therapeutic proteins Draft. 24 September 2015 EMEA/CHMP/BMWP/14327/2006 Rev. 1 Committee for Medicinal Products for Human Use (CHMP). http:// www.ema.europa.eu/docs/en_GB/document_library/ Scientific_guideline/2015/10/WC500194507.pdf. 